\title{
Implementación de catálogo de servicios y servidor KACE
}

\section{Implementation of service catalogue and KACE server}

\author{
ALMANZA-GÓMEZ, Angel Alejandro†*, CORTÉS-CARRILLO, Francisco, MOLINA-DE LA \\ ROSA, Laura y GÓMEZ-VIDAL, Juán Elí
}

\section{Universidad Tecnológica de Chihuahua}

ID $1^{\text {er }}$ Coautor: Angel Alejandro, Almanza-Gómez / ORC ID: 0000-0001-6046-8244, Researcher ID Thomson: G-30892018, CVU CONACYT ID: 802620

\author{
ID $1^{\mathrm{er}}$ Autor: Francisco, Cortés-Carrillo / ORC ID: 0000-0003-4692-6135- Researcher ID Thomson: G-2507-2018, CVU \\ CONACYT ID: 899860
}

ID $2^{\text {do }}$ Coautor: Laura, Molina-De La Rosa / ORC ID: 0000-0003-3571-7098, Researcher ID Thomson: G-3764-2018, CVU CONACYT ID: 172195

ID $3^{\text {er }}$ Coautor: Juán Elí, Gómez-Vidal / ORC ID: 0000-0001-7313-8690, CVU CONACYT ID: 520837

DOI: $10.35429 / J E S C .2019 .8 .3 .32 .49$

Recibido: 08 de Marzo, 2019; Aceptado 26 de Junio, 2019

\section{Resumen}

La problemática se centra en la falta de un sistema de mesa de servicio, así como de escritorio remoto, provocando que el proceso de soporte tenga un periodo de duración más alto del debido y que el departamento de TI no se enfoque en su tarea principal. El no tener las credenciales para algunos equipos de red o servidores, provoca que el funcionamiento óptimo de la red no se logre. Además la falta de actualizaciones a los equipos, hace que estos queden vulnerables a ataques externos. Con esta implementación se pretende lograr una ideal administración de activos de hardware y software, así cómo determinar como está constituida la infraestructura tecnológica de la empresa, esto mediante la creación de un catálogo de servicios que englobe todos y cada uno de los servicios que brinda el departamento, así como la implementación del servidor KACE. Como resultado del proyecto se logra aumentar la productividad del departamento, minimizar el tiempo de soporte, tener plenamente identificada la infraestructura, automatizar el proceso de soporte, generando con esto, una ventaja competitiva para la empresa. Con esto se optimiza el uso de recursos materiales, espacio y seguridad de la información y así mismo se hace mas eficiente el trabajo.

Servidor, Seguridad, KACE

\begin{abstract}
The problem focuses on the lack of a service desk system, as well as a remote desktop system, causing the support process to have a longer period of time than due, and for IT not to focus on its main task. Not having credentials for some network computers or servers, causes optimal network performance to fail. In addition, the lack of updates to computers makes them vulnerable to external attacks. This implementation aims to achieve an ideal management of hardware and software assets, as well as how to determine how the technological infrastructure of the company is constituted, this by creating a catalog of services that encompasses each and every one of the services provided by the department, as well as the implementation of the KACE server. As a result of the Project, it is possible to increase the productivity of the department, minimize the support time, have fully identified the infrastructure, automate the support process, generating with this, a competitive advantage for the company. This optimizes the use of material resources, space and information security, it also makes the work more efficient.
\end{abstract}

Server, Security, KACE

Citación: ALMANZA-GÓMEZ, Angel Alejandro, CORTÉS-CARRILLO, Francisco, MOLINA-DE LA ROSA, Laura y GÓMEZ-VIDAL, Juán Elí. Implementación de catálogo de servicios y servidor KACE. Revista de Ciencias de la Educación. 2019. 3-8: 32-49

\footnotetext{
* Correspondencia del Autor (correo electrónico: aalmanza@utch.edu.mx)

$\dagger$ Investigador contribuyendo como primer autor.
} 


\section{Introducción}

El área de ingeniería y desarrollo de la empresa es de reciente creación, por esta razón este departamento no cuenta con documentación respecto a la infraestructura, servicios o inventarios, es de vital importancia realizar estos procesos para poder tomar control de los recursos tecnológicos de la empresa.

Las contraseñas de los servidores, switches, routers, access points y otros recursos con los que se cuentan, están anotadas en libretas, pizarrones $\mathrm{u}$ hojas sueltas $\mathrm{y}$ no comparten un lugar común, lo que significa que, en caso de requerir una contraseña de manera inmediata, es muy difícil de conseguir. Así mismo no se cuenta con la configuración actual de todos los dispositivos antes mencionados, lo que puede ocasionar que en caso de que sea necesario modificar dicha configuración, el personal no sepa de qué manera actuar.

En cuanto al soporte que se da a los usuarios, actualmente se realiza de manera manual, es decir, el departamento recibe una llamada del usuario, si puede solucionar el problema por teléfono, lo hace, si no, se tiene que ir a su lugar para solucionar el problema, que puede ser; instalar un programa, una actualización o cualquier detalle técnico.

Es por estos motivos se implementará KACE, para dar respuesta a todos esos inconvenientes.

\section{Descripción del método}

\section{Justificación.}

Es de vital importancia recuperar las credenciales de los equipos, así como conocer cómo está constituida la infraestructura tecnológica de la empresa, esto para poder llevar a cabo una correcta administración de los recursos de TI, el catálogo de servicios es el primer paso para ello.

Para esto la implementación del servidor KACE, por todas las funcionalidades y ventajas que este servidor puede generar para facilitar la solución de esta problemática que se lleva a cabo el proyecto.

\section{Planteamiento del problema.}

A falta de un sistema de mesa de servicio, así como de escritorio remoto, el proceso de soporte tiene un periodo de duración más alto de lo que puede ser.

La poca automatización en el proceso de soporte provoca que el departamento de TI no se enfoque como debería en generar las soluciones que requiere la empresa.

El no contar de una manera accesible con las credenciales para los equipos de red o servidores, puede provocar que el funcionamiento óptimo de la red no se logre.

A falta de actualizaciones a los equipos, estos quedan vulnerables a ataques externos, tales como el ransomware.

Por estas razones es necesaria la implementación de este proyecto.

\section{Objetivos}

\section{Objetivo General}

Lograr una ideal administración de activos de hardware y software, así como conocer como está constituida la infraestructura tecnológica de la empresa, mediante la creación de un catálogo que englobe todos y cada uno de los servicios que brinda el departamento, así como la implementación del servidor KACE.

\section{Objetivos específicos}

- Crear un catálogo de servicios.

- Generar una posible ventaja competitiva al implementar un servidor KACE.

- Conocer cómo está construida la infraestructura tecnológica de la empresa.

- Automatizar y minimizar el proceso de soporte a través de una mesa de servicio y de escritorio remoto.

- Automatizar la instalación de programas $\mathrm{y}$ actualizaciones. 


\section{Método}

Análisis de Infraestructura: Se analizará cómo se encuentra la infraestructura de TI y la red, qué equipos son los que se están usando, switches, routers, servidores, sus marcas, modelos, números de serie, así mismo recuperar o reestablecer las credenciales de los equipos y servicios, así como su configuración actual.

Realización de catálogo de servicios: Se documentará toda la información obtenida en el paso anterior, se crearán diagramas de la infraestructura, para tener una referencia más visual de ésta.

Recopilación de información sobre KACE: Investigación a fondo sobre el servidor KACE, para que sirve, como se usa, así como estudiar conceptos básicos para su utilización.

Pruebas de concepto de KACE: Se probará todo lo aprendido durante el paso anterior, creando un laboratorio para probar todas las funciones del servidor, así como para resolver cualquier duda sobre su implementación.

\section{Implementación $\quad$ KACE: $\quad \mathrm{Se}$} implementará el servidor, integrándolo a la red, dejando tiempo para resolver los posibles inconvenientes que se pudieran presentar.

\section{Marco Teórico}

Las herramientas tecnológicas requeridas para el funcionamiento de la empresa y del proyecto son:

\section{Servicio TI}

Es un conjunto de actividades que buscan responder las necesidades de un cliente por medio de un cambio de condición en los bienes informáticos, potenciando el valor de éstos y reduciendo el riesgo inherente del sistema.

\section{Active Directory}

Directorio activo es una estructura jerárquica que almacena, en una base de datos, información sobre redes y dominios. Se utiliza principalmente para obtener información en línea, está diseñado especialmente para entornos de red distribuidos, utiliza protocolos como LDAP, DNS, DHCP y otros.
Almacenamiento (SAN) (Red de área de almacenamiento/Storage Area Network)

Una SAN es una red de almacenamiento integral. Se trata de una arquitectura completa que agrupa los siguientes elementos:

\section{Una red de alta velocidad de canal de fibra o SCSI \\ Un equipo de interconexión dedicado (conmutadores, puentes, etc.) \\ Elementos de almacenamiento de red (discos duros)}

El rendimiento de la SAN está directamente relacionado con el tipo de red que se utiliza.

\section{Backup}

Copias de seguridad es el procedimiento utilizado para hacer copias de información. Se deben realizar sobre los datos más importantes con el propósito que estén disponibles en caso de fallas. Esta debe ser guardada en un soporte de almacenamiento diferente del original, para evitar que un fallo en el mismo pueda estropear el original y la copia.

\section{CCTV. (Circuito Cerrado de TV)}

Probablemente el uso más conocido del CCTV está en los sistemas de vigilancia y seguridad y en aplicaciones tales como establecimientos comerciales, bancos, oficinas gubernamentales, edificios públicos, aeropuertos, etc. El CCTV tiene que estar acompañado de la grabación de los eventos que se vigila con el objeto de obtener evidencia de todos los movimientos.

\section{DHCP (Dynamic Host Configuration Protocol)}

El protocolo de configuración dinámica de host es un estándar TCP/IP diseñado para simplificar la administración de la configuración IP de los equipos de red. Si disponemos de un servidor DHCP, la configuración IP de los PCs puede hacerse de forma automática, evitando así la necesidad de tener que realizar manualmente.

DNS (Domain Name System - Sistema de nombres de dominio)

Un servidor DNS traduce nombres de dominio a IPs y viceversa. En las redes TCP/IP, cada PC dispone de una dirección IP para poder comunicarse con el resto de PCs. 
Además permite averiguar la IP de un PC a partir de su nombre. Para ello, dispone de una base de datos en la cual se almacenan todas las direcciones IP y todos los nombres de las PC pertenecientes a su dominio.

\section{TCP/IP (Transmission Control Protocol -} Protocolo de Control de Transmisión)

Este protocolo define la manera en que se transporta la información desde el dispositivo de origen al dispositivo destino mediante la ejecución por cuatro capas que se encuentran en las tarjetas de red de comunicaciones, iniciando con la capa física, seguido de la capa de red, posteriormente la capa de transporte y por último la capa de aplicación. IP es el protocolo Internet, el cual se ejecuta en la capa de red y permite establecer una dirección física y lógica para conocer la ubicación de los equipos informáticos y establecer la ruta por donde se envía la información.

\section{Firewall}

Es un dispositivo de seguridad de la red que monitorea el tráfico entrante y saliente de red y decide si permite o bloquea tráfico específico en función de un conjunto definido de reglas de seguridad. Un firewall puede ser un programa (software) o un equipo (hardware).

\section{Hipervisor}

Es un programa que permite ejecutar múltiples máquinas virtuales diferentes en un solo hardware. Cada una de estas máquinas virtuales o sistemas operativos serán capaces de ejecutar sus propios programas. En resumen, permite al administrador de TI tener múltiples máquinas virtuales, todas ejecutándose óptimamente en una sola pieza de hardware.

\section{Red Cableada}

Conecta dos o más PCs mediante un cable. También es posible agregar a la red impresoras y otros dispositivos. Para la conexión es necesario un conmutador (switch), que a menudo viene integrado en el router. Sólo las personas autorizadas tienen acceso a los datos compartidos en la red.

\section{Red Inalámbrica}

Una red en la que dos o más terminales se pueden comunicar sin la necesidad de una conexión por cable. Con estas, un usuario puede mantenerse conectado cuando se desplaza dentro de una determinada área geográfica. Por esta razón, se utiliza el término "movilidad" cuando se trata este tema.

\section{Servidor de Archivos}

En el modelo cliente/servidor, un servidor de archivos es un equipo responsable del almacenamiento y administración central de archivos de datos para que otros equipos de la misma red puedan acceder a los archivos. Permite a los usuarios compartir información a través de una red sin tener que transferir físicamente archivos.

\section{SQL Server}

Es un sistema de gestión de bases de datos relacionales (RDBMS) que está diseñado para el entorno empresarial. Esta se ejecuta en T-SQL (Transact -SQL), un conjunto de extensiones de programación de Sybase y Microsoft que añaden varias características a SQL estándar, incluyendo control de transacciones, excepción y manejo de errores, procesamiento fila, así como variables declaradas.

\section{Telefonía IP}

Es una tecnología que permite integrar en una misma red - basada en protocolo IP - las comunicaciones de voz y datos. Muchas veces se utiliza el término de redes convergentes o convergencia IP, aludiendo a un concepto un poco más amplio de integración en la misma red de todas las comunicaciones (voz, datos, video, etc.).

\section{Telefonía Analógica}

Estas líneas pertenecen a la Red de telefonía conmutada (RTC o RTBC) y básicamente están pensadas para transmisión de voz, aunque pueden también transportar datos, por ejemplo, en el caso del fax o de la conexión a Internet ADSL. Se basa en un cable de dos hilos finos de cobre por el cual se transmite una señal eléctrica que se convierte en ondas de sonido. 


\section{Telefonía Digital}

Se trata de un estándar por el cual diversas comunicaciones pueden transmitirse en formato digital (unos y ceros) a la vez a través de los cables de teléfono tradicionales. Forma parte de la Red Digital de Servicios integrados (RDSI o ISDN en inglés), y se basan en un protocolo digital que permite proporcionar una amplia gama de servicios, tanto de voz como de tele servicios y otros tipos.

\section{UTM. (Unified Threat Management)}

Gestión Unificada de Amenazas. Entonces es básicamente un cortafuego de red que engloban múltiples funcionalidades (servicios) en una misma máquina de protección perimetral. Éstos inspeccionan cada paquete (información) que va $o$ viene de Internet (u otra red externa / interna).

\section{VPN. (Virtual Private Network)}

Es una tecnología de red que se utiliza para conectar una o más computadoras a una red privada utilizando Internet. Las empresas suelen utilizar estas redes para que sus empleados, puedan acceder a recursos corporativos que, de otro modo, no podrían.

\section{KACE}

El instrumento de administración de sistemas Dell KACE provee una administración comprensiva de computadoras, servidores, macs, chromebooks, smartphones, tablets, impresoras, dispositivos de networking y otros dispositivos conectados a través de la red.

El dispositivo KACE K1000 es fácil de usar y su arquitectura le permite ser implementado rápidamente. También entrega un amplio catálogo de características para satisfacer las necesidades de administración sistemas de la empresa necesita, desde la implementación inicial de equipos de cómputo como el seguimiento de su administración, seguridad y soporte, sin importar el tamaño de la organización.
Dell KACE K1000 se comunica con los dispositivos gestionados mediante un agente. Este tiene una función bastante importante ya que se encarga tanto de enviar datos del cliente al servidor como de recibir las órdenes que genera el servidor ya sea para realizar una instalación, un inventario, entre otras más funciones.

Este agente utiliza el protocolo de mensajería AMP (Agent Messaging Protocol), y es ejecutado en el dispositivo cliente como un servicio. El cliente con agente instalado se comunica con el servidor K1000 a través de TCP/IP utilizando el puerto de comunicación 52230.

Cuando el servidor K1000 envía o recibe paquetes lo hace a través del puerto de distribución 80 (http) o 443 (SSL) si está habilitado.

Entre muchas de sus características Dell KACE K1000 tiene la capacidad de detectar automáticamente todos los dispositivos conectados a la red y el software instalado, ya sea en dispositivos con el agente instalado para su posterior administración o sin agente con el fin de mantener el inventario informativo completo, además administra los activos no informáticos desde la implementación hasta su retirada.

\section{Agentes Inteligentes}

Es una pieza de software que ejecuta una tarea dada utilizando información recolectada del ambiente, para actuar de manera apropiada hasta completar la tarea de manera exitosa. Debe ser capaz de auto ajustarse basándose en los cambios que ocurren en su ambiente de forma.

Parches de aplicaciones y software

Consta de cambios que se aplican a un programa, para corregir errores, agregarle funcionalidad y/o actualizarlo. A menudo consiste en una actualización del archivo ejecutable de un programa. En este caso, el archivo binario es modificado para añadir los cambios o ser completamente reemplazados. 
Instalación Administrada. (MI)

Son el mecanismo principal para la implementación o la eliminación de aplicaciones de los dispositivos administrados. Cada una describe el título y la versión de la aplicación específica que se instalará o se eliminará, incluidos los comandos de instalación, los archivos de instalación y los dispositivos de destino (identificados por etiqueta).

\section{Wake on LAN}

Es un protocolo que permite encender un equipo de forma remota. Este es posible gracias a la adopción del modelo "muy bajo consumo" de los dispositivos actuales que, cuando están apagados, si están conectados a la corriente siguen haciendo uso de algunas funciones muy básicas con un consumo eléctrico prácticamente inapreciable. Hoy en día, prácticamente cualquier placa base moderna es compatible con este protocolo.

\section{Scripts}

Proporcionan una interfaz interactiva para realizar tareas que, en general, requieren un proceso manual o de programación avanzada. Se pueden crear scripts y ejecutarlos para realizar tareas en dispositivos de destino a través de la red.

\section{LDAP (Lightweight Directory Access Protocol)}

El Protocolo ligero de acceso a directorios es un conjunto de protocolos abiertos usados para acceder información guardada centralmente a través de la red. Está basado en el estándar X.500 para compartir directorios, pero es menos complejo e intensivo en el uso de recursos, que contiene información de forma jerárquica y categorizada, que puede incluir nombres, directorios y números telefónicos.

\section{Mesa de Servicio}

Permite al departamento de TI responder incidentes inesperados dentro de la infraestructura de tecnología o los servicios que provee. Tiene la capacidad de respuesta y reacción ante cualquier incidente, de manera rápida, llevando un registro y control de los mismos.

\section{OVAL}

Es un estándar internacional de seguridad de la información que promueve el contenido de seguridad y estandariza la transferencia de esta información en todos los servicios y herramientas de seguridad. El lenguaje OVAL es un grupo de esquemas XML que se utiliza para representar la información del sistema expresando estados específicos de la máquina, y notificando los resultados de la evaluación.

\section{Etiquetas}

Son contenedores que permiten organizar y clasificar elementos, como dispositivos, para que se puedan administrar como un grupo. Pueden asignarse manualmente a elementos específicos o asignarse automáticamente a elementos cuando están asociadas con criterio, tales como las consultas SQL o LDAP.

\section{Desarrollo}

\section{Análisis de la infraestructura}

\section{Servicio de Active Directory}

El servicio de active Directory se encuentra alojado en una máquina virtual dentro del servidor de virtualización Dell PowerEdge R710, y cuenta con la dirección IP 192.XXX.10.1. Éste depende de un switch TPLink, al cual está conectado en los puertos 1 y 2 , un switch Dell, al cual está conectado por los puertos $17,19,21$ y 23 , esto ya que el servidor R710 contiene múltiples máquinas virtuales y todas necesitan tener una salida hacia la red. El servicio depende también de la SAN Equallogic, que es el servidor de almacenamiento (Storage Area Network), en donde se encuentra alojada la imagen del sistema operativo en el cual corre active Directory. A su vez, otra de las dependencias de este servicio, son las UPS ISB y Tripp lite, el UTM Cyberoam y el router que le da salida a internet.

Este servicio no cuenta con credenciales, ya que, solo los usuarios administradores pueden acceder a él con sus propias credenciales.

El servicio de Active Directory se maneja a través de dos principales unidades organizativas, la OU 20, y la OU C, éstas hacen referencia a la matriz y a la sucursal, respectivamente. 
A su vez, la OU 20 contiene otras 9 unidades organizativas, la cuales son: administración, almacen, compras, dirección, mostrador, printers (Impresoras), ingeniería, soporte y ventas, y cada una de ellas contiene a los usuarios de dichas áreas. Dado que la OU C es sucursal, solo contiene una unidad organizativa, la cual es ventas.

Anexo \#1, se muestra la tabla del servicio de active Directory.

Anexo \#2, Se muestra la configuración de Active Directory.

Anexo \#3, Diagrama Visio de arquitectura del servicio.

\section{Servicio de Almacenamiento}

El servicio de almacenamiento se encuentra alojado en la SAN Dell Equallogic PS4000E, cuenta con una interfaz gráfica web SAN Head Quarters a la cual se accede por medio de la ip 192.XXX.10.14 con las credenciales de usuario "grpadmin" y el Password "grpadmin". Este servidor cuenta con 15 discos duros SATA de $500 \mathrm{~GB}$ agrupados por medio de raid tipo 6, lo que da un espacio crudo de $7 \mathrm{~TB}$ pero que con la configuración de RAID solo deja disponibles 5.66 TB, el espacio utilizado a la fecha de la realización de la hoja de servicio es de $2.68 \mathrm{~TB}$ y cuenta con 2 tarjetas NIC.

Cabe destacar, que, aunque el sistema cuenta con 15 discos duros, estos se encuentran incluidos en un arreglo RAID 6, el cual engloba todos los discos duros en un solo virtual, y a partir de ahí, se crean volúmenes para guardar diferente información.

Para su funcionamiento, este equipo solamente depende de los UPS ISB y Tripp-Lite.

Las imágenes con el desglose de los discos duros y volúmenes de almacenamiento del sistema:

Anexo \#4, Configuración del Almacenamiento

Anexo \#5 Configuración del Almacenamiento 2

El sistema cuenta con un procedimiento de apagado especial, ya que, si es apagado sin seguir este procedimiento, se puede causar un daño al arreglo RAID:
1. Iniciar la aplicación web, ingresando el siguiente link: http://192.XXX.10.14

2. En el menú Group, dirigirnos a la opción "members" dentro del grupo que queremos apagar.

3. Seleccionar el miembro Samsara.

4. Dirigirnos a la pestaña Maintenance y dar clic en el botón "Shut down".

5. Después de que se ejecute el comando, es necesario apagar manualmente las dos fuentes de poder.

Anexo \#6 Servicio Almacenamiento Anexo \#7 Configuración Almacenamiento 3 Anexo \#8 Diagrama del servicio de almacenamiento

\section{Servicio de Backup}

El servicio de Backup es llevado a cabo por medio del software Veeam Free Edition, y se realiza en un disco duro externo Adata de $2 \mathrm{~Tb}$ y en un disco duro interno virtual de $1 \mathrm{~TB}$. La única dependencia de este servicio es el servidor de virtualización PowerEdge R710, en donde se encuentra alojada la máquina virtual Windows server que corre el software de respaldo.

El respaldo interno de las máquinas virtuales de ERP, APPS y COMPAC se lleva a cabo cada día a las 3:00 A.M. y se guarda en la ruta "V:IVM'S Backup", mientras que el externo, se lleva a cabo una vez por semana, los sábados a las 3 de la tarde en la ruta "F:IVM'S Backup".

\section{Anexo \#9 Servicio Backup}

\section{Servicio de CCTV}

Está constituido por 15 cámaras y un DVR marca DAHUA modelo HS-1604-LE-A para grabación, el cual está ubicado en el site y al que se puede acceder a través de la IP 192.XXX.10.15 por el puerto 8000 con las credenciales "adming" y el Password "ing20N".

Este depende del switch DELL PowerConnect 2848 que está conectado por el puerto número 24, depende también del Patch Pannel, del UTM y de un PDU, ya que en dicho site, no existe ningún UPS.

Anexo \#10 Servicio de CCTV

Anexo \#11 Diagrama de CCTV 


\section{Servicios DHCP y DNS}

Dado que éstos no consumen muchos recursos, estos se encuentran ubicados en una misma máquina virtual Windows Server 2012 alojada en el servidor DELL PowerEdge R710, con la ip 192.XXX.10.2. Al estar alojados en el mismo servidor de virtualización que otros servicios mencionados anteriormente, las dependencias y los puertos por los que están conectados son los mismos y no necesitan credenciales.

El servicio de DNS cuenta con una zona de búsqueda directa, en la que se encuentran registrados todos y cada uno de los equipos de cómputo de los usuarios, así como servidores, máquinas virtuales e impresoras. De la misma manera, cuenta con una zona de búsqueda inversa, la cual tiene registro de los servidores, máquinas virtuales, la DVR y el UTM.

\section{Anexo \#12 Servicio DNS 1}

Anexo \#13 Servicio DNS 2

El DHCP cuenta con un conjunto de direcciones IP, de la 192.XX.10.50 a la 192.XXX.10.150, dejando 100 direcciones IP para repartir a los equipos de los usuarios y visitantes, con excepción de ciertas IP que se encuentran excluidas de esta distribución, esto se debe a que dichas direcciones están casadas con impresoras o servidores que necesitan tener una IP fija.

Existen 2 direcciones reservadas, la del enrutador que brinda salida a internet, y la del servidor de DNS.

\section{Anexo \#14 Servicio DHCP}

\section{Servicio Hipervisor}

Este se encuentra alojado en el servidor Dell PowerEdge R710, con la IP 192.XXX.10.1, que cuenta con 96 GB de RAM, la cual está repartida entre las diferentes máquinas virtuales que este contiene. Además tiene un disco duro de 584 $\mathrm{GB}$, aunque las máquinas virtuales no se encuentran ubicadas en este almacenamiento, sino en el servidor SAN de almacenamiento.

Este servicio depende de las UPS ISB y Tripp Lite, así como del servidor SAN Equallogic, y cuenta con las credenciales "Administrador" con contraseña “C0xxx@ms@r4”.
El servidor de virtualización cuenta actualmente con 10 máquinas virtuales, listadas de la siguiente manera:

Anexo \#15 Servicio Hipervisor

\section{Servicio Red Cableada}

Es de vital importancia para la empresa, ya que, sin este, ninguno de los otros servicios podría ser entregado. Cuenta con 2 site, en uno se encuentran todos los servidores, el UTM, un Switch Dell X1026, un switch TP-Link TLSG1024, así como el modem de internet de Total Play.

En el otro, se encuentran ubicados el DVR, el modem de internet de Telmex, un switch PowerConnect 2848 y el patch panel del cual salen todas las conexiones de los nodos que se encuentran en la empresa. Los switches anteriormente mencionados son los equipos principales y vitales para este servicio, a continuación, una descripción de cada uno de ellos.

El switch Dell X1026 es un switch gigabit ethernet de 24 puertos, cuenta con la IP 192.XXX.10.11, depende solamente del UPS Tripp Lite. Los puertos 7, 17, 19, 21 y 23 están conectados al servidor de virtualización, el puerto número 8 está conectado al conmutador KX-TDE100, el puerto 20 está conectado al UTM y los puertos 22 y 24 están conectados al servidor de almacenamiento. Como se puede apreciar, este switch es el que crea la comunicación entre los servidores, máquinas virtuales y el almacenamiento de red.

El switch TP-Link TL-SG1024 es un switch gigabit ethernet de 24 puertos, no cuenta con una IP ya que no es administrable, este sirve solo como un puente entre los servidores de almacenamiento (conectado a través de los puertos $9,10,11$ y 12) y el de virtualización (conectado a través de los puertos 1 y 2 ).

El Switch DELL PowerConnect 2848 es un switch gigabit ethernet de 48 puertos, cuenta con la IP 192.XXX.10.12, éste es el switch principal de la red cableada, ya que está conectado al patch panel que reparte la red en el edificio, además, está conectado directamente al DVR por el puerto número 24 para grabación de las cámaras de circuito cerrado de televisión. 
Se muestra la hoja del servicio de red cableada, así como el diagrama de arquitectura de la red en:

\section{Anexo \#16 Servicio Red Cableada Anexo \#17 Diagrama Red Cableada}

\section{Servicio Red Inalámbrica.}

Consta de un único access point marca ubiquiti modelo UAP-AC-PRO. Éste cuenta con una interfaz de configuración web Unifi Controller a la cual se puede acceder por medio de la IP 192.XXX.10.252, se encuentra conectado a un nodo de internet ubicado en el site principal. Dicho access point radía una señal en $2.4 \mathrm{GHz}$ y $5 \mathrm{GHz}$ con el SSID SAMSARA y el Password “*S@m\#W1F1@XXX7”. La única dependencia de este servicio, reside en el switch power connect, pues éste es el que lleva el internet hasta el nodo al cual está conectado el AP.

Anexo \#18, Servicio de Red Inalámbrica Anexo \#19 Diagrama de Red Inalámbrica

\section{Realización del catálogo de servicios}

¿Por qué es importante el catálogo de servicios? Un Catálogo de Servicios TI describe cada servicio con sus características. No importa si un usuario necesita solicitar el acceso a una aplicación, la recuperación de un backup o de una cuenta de usuario, el Catálogo de Servicios es el punto de acceso al conjunto de productos y servicios que le ofrece el departamento de TI, facilitándole el acceso a esta información y a su tramitación.

La sistematización como método proporciona una aproximación analítica a los procesos que se desarrollan en una organización. Lleva implícito su ordenamiento. Por tanto, el esfuerzo realizado en las actividades de registro y de clasificación va a posibilitar el acceso, el control y el manejo ágil de la información.

El proceso de la creación del catálogo de servicios de la empresa, comprende de la realización de una hoja de Excel con todos los datos de cada uno de los servicios, dichos datos son:

\footnotetext{
- Nombre del servicio

- $\quad$ El equipo en el que reside el servicio

- Dependencias (¿De qué otros equipos depende este servicio?)

- Credenciales

- Configuración Actual
}

\section{Diagrama de Visio con la arquitectura del} servicio

\section{Recopilación de información sobre KACE}

El instrumento de administración de sistemas Dell KACE provee una administración comprensiva de computadoras, servidores, macs, chromebooks, smartphones, tablets, impresoras, dispositivos de networking y otros dispositivos conectados a través de la red.

El dispositivo KACE K1000 es fácil de usar y su arquitectura le permite ser implementado rápidamente. También entrega un amplio catálogo de características para satisfacer las necesidades de administración sistemas de la empresa necesita, desde la implementación inicial de equipos de cómputo como el seguimiento de su administración, seguridad y soporte, sin importar el tamaño de la organización.

Dell KACE K1000 se comunica con los dispositivos gestionados mediante un agente. Este tiene una función bastante importante ya que se encarga tanto de enviar datos del cliente al servidor como de recibir las órdenes que genera el servidor ya sea para realizar una instalación, un inventario, una actualización, entre otras funciones más.

Este agente utiliza el protocolo de mensajería AMP (Agent Messaging Protocol), y es ejecutado en el dispositivo cliente como un servicio. El cliente con agente instalado se comunica con el servidor K1000 a través de TCP/IP utilizando el puerto de comunicación 52230. Cuando el servidor K1000 envía o recibe paquetes lo hace a través del puerto de distribución 80 (http) o 443 (SSL) si está habilitado.

Entre muchas de sus características Dell KACE K1000 tiene la capacidad de detectar automáticamente todos los dispositivos conectados a la red y el software instalado, ya sea en dispositivos con el agente instalado para su posterior administración o sin agente con el fin de mantener el inventario informativo completo, además administra los activos no informáticos desde la implementación hasta su retirada. 


\section{Pruebas de concepto KACE}

\section{Configuración Inicial}

\section{Figura 2, Panel de Control de KACE}

EL dispositivo de administración KACE K1000 es configurado de la siguiente manera:

Dirección ip estática 192.XXX.10.16 con una máscara de subred 255.XXX.255.0, y se une al dominio SOLUCIONES. local (el dominio local), se encuentra conectado a los puertos $1 \mathrm{y}$ 2 del switch TP-Link así como a los puertos 17, 19, 21 y 23 del switch Dell, ubicados en el site principal, así mismo depende del servidor SAN Equallogic, el UTM Cyberoam, el router de total play y las UPS ISB y Tripp Lite, se ingresa el nombre de la compañía de la siguiente manera: XXXXXXX TECNOLOGIA Y SOLUCIONES, con el mail de administrador Gxxxx.rxxxxxx@xxxxxxxxxx.com,y se usan las credenciales admin con Password "K4c3kuv0!", tiene como servidor de DNS el servidor local (192.XXX,10.2) para poder resolver su propio nombre.

En el servidor local de DNS se agrega la entrada del nombre del servidor KACE de la siguiente manera:

Se crea un nuevo registro en la zona de búsqueda directa del servidor DNS y se selecciona el tipo de registro Host (A o AAAA).

IFigura 3, Crear nuevo registro en DNS Se agrega el nombre por el cual se desea ingresar al sistema, el FQDN (Fully Quallified Domain Name), el cual es el nombre asignado más el nombre del dominio, lo que resulta en el nombre xxxxxxxk1k.soluciones.local, y, por último, se agrega la dirección IP la cual se desea traducir al nombre antes mencionado.

Figura 4, Crear nuevo registro en DNS 2

De esa manera, se puede ingresar al servidor KACE con tan solo teclear en el navegador la dirección: xxxxxk1k/adminui.
Cabe destacar que el servidor kace tiene múltiples interfaces de usuario, la de administrador la cual sirve para administrar todos los servicios que este servidor es capaz de proveer, la de sistema, que funciona para manejar las opciones del sistema como dirección ip, almacenamiento, etc. Y la de usuario, en la que los usuarios pueden levantar tickets en la mesa de servicio o descargar archivos o instaladores almacenados en el servidor KACE.

\section{Implementación KACE}

Instalación del agente.

Para instalar el agente inteligente de KACE en los dispositivos a administrar se tienen que llevar acabo los siguientes pasos:

Abrir puertos necesarios para la administración remota de KACE. Abrir el firewall de Windows, ir a seguridad avanzada y en esa pantalla ordenar las reglas por nombre de la siguiente manera:

Windows 1

Figura 5, Reglas del Firewall de Filtrar las reglas por perfil de dominio: Windows 2

Figura 6, Reglas del Firewall de

Habilitar las reglas de administración remota, con excepcipón de las de firewall, esto ya que esto supondria un riesgo de seguridad, al dejar que el firewall sea administrado de manera remota. Windows 3

lustración 7, Reglas del Firewall de

Activar las reglas de compartir archivos, con excepción de la de ICMPv6, esto ya que no se esta manejando IPv6.

Windows 4

Figura 8, Reglas del Firewall de 
Abrir administración de equipos (click derecho sobre el icono de Windows > Administración de equipos) click en servicios y aplicaciones y entrar a la opción Control WMI, click derecho, propiedades y dirigirse a la pestaña seguridad, luego click sobre el botón seguridad, y se agrega el grupo SOL-DLINGENIERIA, activando los permisos para ejecutar métodos, escritura completa, escritura parcial, escritura con proveedor, habilitar cuenta, llamada remota habilitada y seguridad de lectura, esto da permisos al grupo de ingeniería en active directory para poder administrar equipos en la red de manera remota.

Además de los pasos anteriores, se crea un usuario para el KACE en active directory, y se agrega a los usuarios de administración remota, lo que permite que KACE se conecte de manera remota a otros ordenadores. En este caso, el usuario agregado es XXXX_USER.

Ejecutar el servicio de administración remota en el dispositivo a administrar de la siguiente manera:

Abrir el símbolo del sistema como administrador y teclear el siguiente comando: winrm xxxxxconfig. De esta manera se activa el servicio de administración remota.

Figura 9, Activar Servicio de Administración Remota

Activar la detección de redes y el uso compartido de archivos e impresoras:

Figura 10, Activar la detección de redes y el uso compartido de archivos e impresoras

Deshabilitar UAC:

\section{Figura 11, Deshabilitar UAC}

Abrir el explorador de archivos y teclear xxxxxk1k/agentprovisioning/Windows, esto abre la carpeta compartida de KACE, de donde se descarga el agente inteligente. Descargarlo e instalarlo siguiendo el wizard de configuración. Una vez instalado el agente, correr el siguiente comando en el símbolo del sistema, como administrador:

"C:IProgram Files
Dell\KACElrunkbot.exe" -s 40

$(\mathrm{xxx})$
De esta manera, el inventario de KACE corre en la computadora, y ahora puede ser administrada desde KACE.

\section{Actividades Complementarias}

Instalación Uvnc

Como parte de una solución integral, se instala uvnc para manejo de escritorio remoto, y de esta manera, agilizar el proceso de soporte a los usuarios. Para instalar uvnc se llevan a cabo los siguientes pasos:

Abrir el wizard de instalación de uvnc, palomear las opciones registrar ultravnc server como un servicio del sistema, iniciar o reiniciar el servicio de ultravnc, dar click en siguiente e instalar.

Figura 12, Instalación uVNC 1

Figura 13, Instalación uVNC 2

Una vez instalado, se configura con los siguientes passwords:

VNC Password: R3x0xxxdm!

View-Only Password: R3x0xxx1w

Se palomea la opción requerir login de Microsoft, y se agrega el grupo de dominio Soldl-ingeniería y el grupo local de administradores, esto hace que el grupo de ingeniería y el usuario administrador local puedan utilizar el escritorio remoto de uvnc.

Figura 14, Instalación uVNC 3

Una vez ejecutados los pasos anteriores, KACE ya se encuentra administrando por completo los equipos de cómputo de la red, cabe destacar que hay que llevar a cabo los pasos anteriores en cada uno de los equipos que se administrarán, a partir de este momento, KACE es capaz de instalar software de manera remota y masiva, apagar o encender equipos, ejecutar scripts o instalar parches. 


\section{Resultados y análisis}

\section{Resultados cuantitativos}

En base al análisis de la situación previa a la realización de este proyecto, y al análisis una vez desarrollado el proyecto, podemos llegar a las siguientes conclusiones y resultados. Al tener en cuenta la gran tarea de gestión de los dispositivos End Point de la empresa y del continuo crecimiento de estos, se requiere de la implementación de Kace de quest, servidor que permite administrar cada una de las distintas funciones de los dispositivos.

Se deben mencionar los numerosos efectos favorables que son producto de esta implementación comenzando por el primer obstáculo presentado que es la falta de claridad y control del número de Pc y Laptops a nivel hardware y Software (Control de licencias) al igual que el uso de bastante tiempo en las tareas manuales de administración repetitiva de software.

Cuando una serie de dispositivos se adquieren por la empresa se requiere de la identificación, documentación y registro de ellos para controlar la entrada. Esto involucra un trabajo extenso de revisión y almacenamiento de información y se requiere de una gran cantidad de tiempo. Kace descubre y registra como inventario todas las Pc y Laptops conectados a la red, identificando y documentando su Hardware y Software lo cual finaliza en el ahorro del 50$80 \%$ de tiempo de realización de estas tareas.

De manera lógica se requiere de la inversión para la implementación del servidor y cuando esta ocurre se requiere saber de qué modo se realizará el retorno de la inversión. Existen diversas calculadoras de este retorno y al obtener los resultados más de tres cuartos de los clientes de KACE mencionan que la aplicación se retribuye en seis meses o menos.

\section{Resultados cualitativos}

La aplicación de esta solución nos está otorgando diversos desenlaces para nuestros problemas dados. Hemos de ver que se presentan distintos de estos problemas como el inventariado de los recursos de hardware y software, así como el riesgo por incumplimiento de licencias ante organismos tales como BSA (Business Software Alliance) y el IMPI (Instituto Mexicano de Propiedad Intelectual).
Además, en muchas organizaciones los usuarios instalan aplicaciones fuera de política, en sus Pc's y Laptop's, así como extraen información por medio de los puertos de salida de los dispositivos (USB, DVD).

En las soluciones que automáticamente van surgiendo se comienza a observar el alcance que tienen para cada campo requerido, por ejemplo, todos y cada uno de los dispositivos finales que se emplean como pcs, laptops, macs, servidores, tabletas, impresoras, etc. Al igual que dispositivos que no requieren adquisición de licencia tales como ciertas impresoras, lectores de códigos de barras, monitores, etc. sus diversos sistemas operativos como Windows, ios, unix, Linux y Android. Este alcance es claramente visible puesto que se llega a cada uno de los elementos que estén en un compendio que una empresa tenga.

Una vez mencionado el alcance físico que se tiene, se incorpora el alcance del entorno intangible con la identificación y la documentación del software instalado y su licencia de cada pc o laptop. Para el administrador de sistemas: Identifica las IP, Nombre del Dominio, Estado de conexión, BIOS, procesador, disco y memoria, así mismo agentes o programas instalados no deseados.

Revisemos que también hace una actualización de sistema operativo y aplicaciones (parches). El no tener estas actualizaciones aumenta el riesgo de ataques cibernéticos (virus, hackers, RANSOMWARE, etc.).

Recordemos ciertas de los apuros que se presentan a la hora de trabajar con estos dispositivos finales. Se requiere una inversión enorme de tiempo y demora en la instalación manual de nuevas aplicaciones o software adquirido por la organización. Se está obligada la atención a requerimientos de soporte técnico por usuarios (Tickets de Servicio), gran parte de ellos debido a la falta de políticas y control de accesos.

Levantamiento de inventario físico en hardware y software de manera manual (hojas de Excel). Y finalmente la incertidumbre de si el equipo se encuentra dentro de garantía o no, así como desconocimiento del número de serie del equipo, nombre del proveedor y fecha de compra.

ALMANZA-GÓMEZ, Angel Alejandro, CORTÉS-CARRILLO, Francisco, MOLINA-DE LA ROSA, Laura y GÓMEZ-VIDAL, Juán Elí. Implementación de catálogo de servicios y servidor KACE. Revista de Ciencias de la Educación. 2019 
El servidor Kace es una herramienta que otorgara la actualización automática y programada de las nuevas versiones de los sistemas operativos y aplicaciones de Microsoft y otros fabricantes de Software, lo cual elimina la búsqueda manual de las actualizaciones. El servidor coloca una distribución o instalación centralizada de aplicaciones nuevas desde la consola del administrador con la que por supuesto se mantiene una constante comunicación.

Al interactuar con esta herramienta se pueden encontrar diferentes tipos de administrador, los cuales por supuesto manejan métodos o procedimientos diferentes y es por ello que si él no encuentra cierta petición se cuenta con una mesa de ayuda, donde los usuarios pueden levantar requerimientos de soporte y dar seguimiento al proceso de atención, configurando el acuerdo de nivel de servicios. En ella el objetivo principal es responder de una manera oportuna, eficiente y con alta calidad a las peticiones que dichos usuarios realicen, en relación a los diversos aspectos de la Tecnología de la Información.

Contando con lo anterior se le permite al administrador de sistemas entregar reportes del inventario de Hardware y Software a la dirección de la organización de forma eficiente y confiable en el tiempo convenido. Al igual que permite conocer el tiempo que tiene el equipo en la organización, vigencia de garantía, apoyando esto a sus planes de renovación tecnológica de acuerdo a las políticas de la organización.

Hablando del licenciamiento que se debe tener de base, KACE identifica y reporta el Software instalado en los equipos contra las licencias adquiridas por la organización, el informe del porcentaje de uso de las aplicaciones por cada usuario ayuda a identificar, que aplicaciones no son usadas por los usuarios y sin embargo la organización paga el licenciamiento anual de estas aplicaciones. De esta manera los administradores determinan en la siguiente renovación de software si compran o no la licencia y descartando las que ya no son requeridas. Por ende, se generarán ahorros impresionantes en la renovación de licencias. (ROI, retorno de inversión).
Además, esta solución gobierna y administra el dispositivo del usuario. Se implementan una serie de controles de confianza y seguridad como por ejemplo el bloqueo de instalación de aplicaciones o elementos fuera de política informática de la organización fungiendo como filtro de información innecesaria a través de un acceso remoto de los administradores de sistemas a los equipos. Se otorga un bloqueo de puertos de salida de forma centralizada.

Al estar situadas todas estas ventajas se comienzan a reforzar las políticas de instalación y distribución de información, así como los elementos que no están indicados o que pueden ser antirreglamentarios.

Se origina la estandarización de la configuración de la empresa por supuesto al llevar un mejor control de todos estos dispositivos mencionados, y así se evita ir equipo por equipo actualizando software $\mathrm{y}$ sistemas. Así la administración de Parches y distribución de Software se hace de manera más fácil o en ocasiones de manera automática.

De los equipos se pueden realizar tareas técnicas de manera automatizada como: contar con accesos Móviles para Detectar el equipo aun estando conectado fuera de la RED. Tener el control Remoto para evitar que los especialistas tengan que trasladarse para resolver problemas. Asignación de un monitoreo y alerta para anticipar riesgos, mal funcionamiento, malware, vencimiento de garantías, etc. Contar con un portal del usuario y un autoservicio, esto para enviar y dar seguimiento a solicitudes de servicio. Se cuenta con la Mesa de Servicio mencionada para Reportar y generar estadísticas de incidencias y Niveles de Servicio (tiempo en que es atendida una incidencia de requerimiento de soporte).

\section{Conclusiones}

Como un conjunto de atributos se nos presenta esta solución para implementar en la empresa en la que se perciben todo tipo de necesidades. Se comienza a simplificar el entendimiento de la administración de los dispositivos y de los requerimientos que tienen. Revisemos que en cada uno se puede administrar los privilegios es decir delimita quien recibe cierta cantidad de permisos. 
Con un escaneo de vulnerabilidad se pueden obtener fallas en la seguridad a nivel equipo. KACE Hace más eficiente el soporte y Servicio a Usuarios. Sencillamente se obtienen una serie de facilidades de operación para los usuarios primarios y finales de la empresa. Se determina que KACE ayuda a que los líderes del área de sistemas, pasen de una aportación operativa a una aportación estratégica y se ubiquen en el plano de proponer mejoras a los procesos a través de las tecnologías de información y de esta manera que sumen a que los directivos propongan y realicen el cambio esperado, de acuerdo a la visión.

\section{Agradecimiento}

A la empresa por dar las facilidades de espacio y técnicas para poder desarrollar este proyecto y a la Universidad por asignar el tiempo necesario a los docentes para apoyar este proyecto en el aspecto académico y técnico.

\section{Referencias}

(s.f.).

Obtenido

https://support.microsoft.com/eses/help/196464

(s.f.).

Obtenido

de

http://es.ccm.net/contents/638-san-red-de-areade-almacenamiento

(s.f.).

Obtenido de http://www.eyca.com.mx/funcionamiento-cctv

(s.f.).

Obtenido

de

http://www.ite.educacion.es/formacion/material es/85/cd/linux/m2/servidor_dhcp.html

(s.f.).

Obtenido

de

http://www.ite.educacion.es/formacion/material es/85/cd/linux/m2/servidor_dns.html

(s.f.).

Obtenido

de

http://www.cisco.com/c/es_mx/products/securit $\mathrm{y} /$ firewalls/what-is-a-firewall.html

(s.f.).

Obtenido

de

https://www.xataka.com/especiales/maquinasvirtuales-que-son-como-funcionan-y-comoutilizarlas

(s.f.).

Obtenido de http://www.informaticamilenium.com.mx/es/te mas/cuales-son-los-servicios-it.html (s.f.).

Obtenido

de https://www.mindomo.com/es/mindmap/redes17f643a4643b4b9ba3a64ddcaed42343

(s.f.).

Obtenido

de

http://es.ccm.net/contents/818-redes-

inalambricas

(s.f.).

Obtenido

de

http://searchdatacenter.techtarget.com/es/defini cion/Servidor-de-archivos

(s.f.).

Obtenido

de

http://searchdatacenter.techtarget.com/es/defini cion/Sistema-de-gestion-de-bases-de-datosrelacionales-RDBMS

(s.f.). Obtenido de http://www.centralitastelefonicasip.net/voip

(s.f.). Obtenido de http://www.tpartner.net/2015/11/26/telefoniaanalogica-vs-digital-vs-ip-que-tecnologiaelegir-para-la-empresa/

(s.f.). Obtenido de http://www.ingdiaz.org/quees-un-firewall-utm/

(s.f.). Obtenido de https://www.welivesecurity.com/laes/2012/09/10/vpn-funcionamiento-privacidadinformacion/

(s.f.).

Obtenido de https://www.tecnoquest.com/blogtecnoquest/item/43-gestion-de-sistemassimplificada-dell-kace

(s.f.). Obtenido de http://concyteg.gob.mx/ideasConcyteg/Archivo s/31072008_QUE_SON_AGENTES_INTELIG ENTES_SOFTWARE.pdf

(s.f.). Obtenido de http://trimestralconfiguracion.blogspot.mx/p/act ualizacion.html

(s.f.). Obtenido de https://www.testdevelocidad.es/redes/wake-onlan-utilizarlo/ 
(s.f.).

Obtenido

de

http://docadmin.quest.com/k1000-as-a-

service/7.1/gu\%C3\%ADa-de-

administraci\%C3\%B3n/implementaci\%C3\%B3

n-de-paquetes-en-dispositivos-

administrados/ejecuci\%C3\%B3n-de-scripts-en-

dispositivos-administrados/acerca-de-los-scripts

(s.f.). Obtenido de http://web.mit.edu/rheldoc/4/RH-DOCS/rhel-rg-es-4/ch-ldap.html

(s.f.). Obtenido de http://www.manageengine.com.mx/Blog/Mesade-ayuda-o-mesa-de-servicios-cual-es-ladiferencia.html

(s.f.).

Obtenido

de https://www.ibm.com/support/knowledgecenter /es/SS6MCG_8.2.0/com.ibm.tem.doc_8.2/Secu rity_and_Compliance/SCAP_Users_Guide/c_o pen_vulnerability_and_assessm.html

(s.f.).

Obtenido

de

http://docadmin.quest.com/k1000-as-a-

service/7.1/gu\%C3\%ADa-de-

administraci\%C3\%B3n/introducci\%C3\%B3n/c onfiguraci\% $\% 3 \% \mathrm{~B} 3 \mathrm{n}$-y-uso-de-etiquetas-paraadministrar-grupos-de-elementos/acerca-de-lasetiquetas

\section{Anexos}

\section{Configuración Inicial}

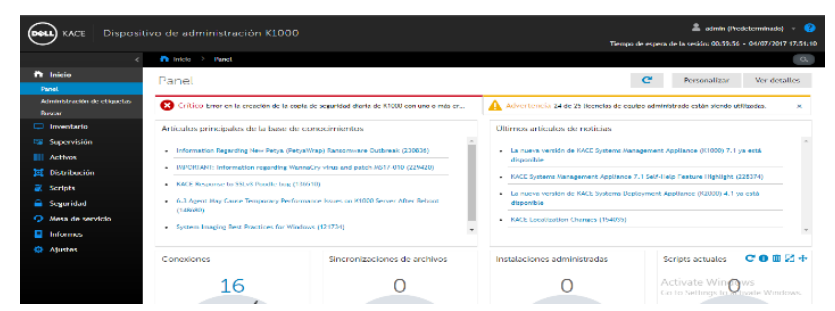

Figura 1, Panel de Control de KACE

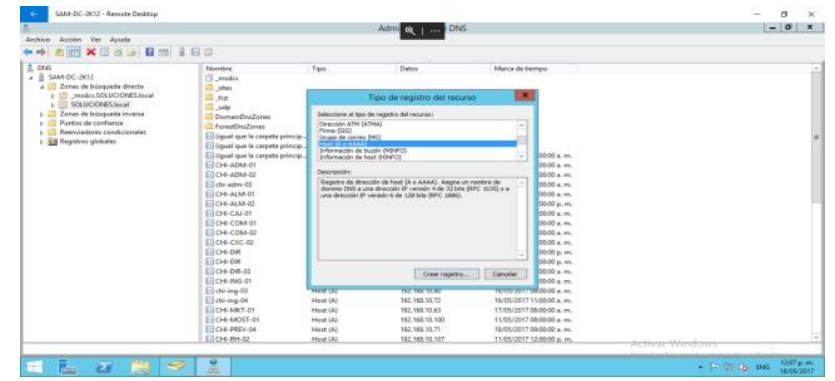

Figura 2, Crear nuevo registro en DNS

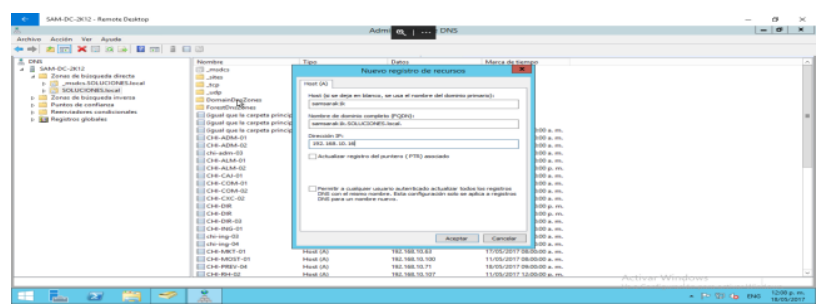

Figura 3, Crear nuevo registro en DNS 2

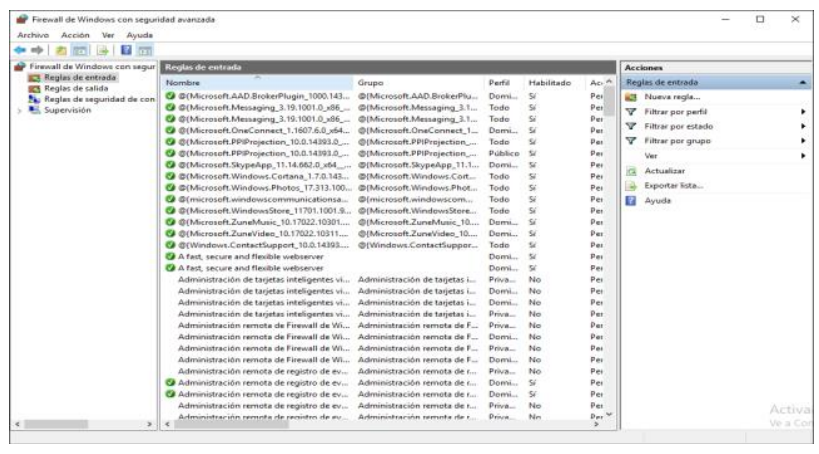

Figura 4, Reglas del Firewall de Windows 1

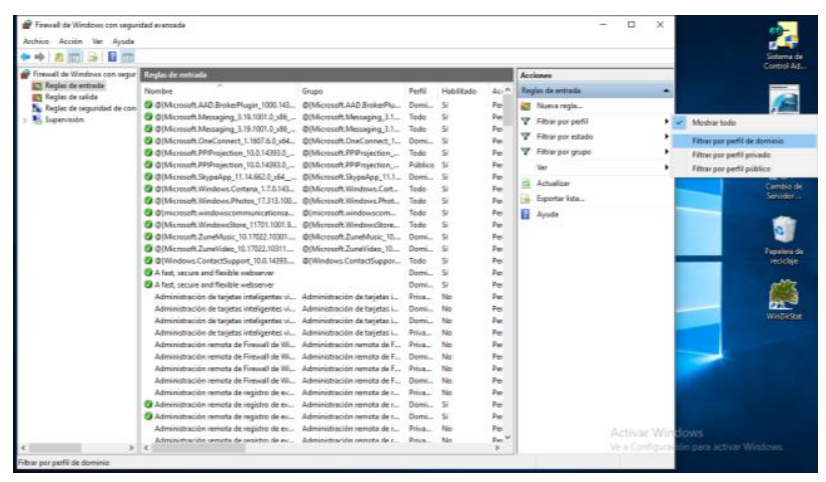

Figura 5, Reglas del Firewall de Windows 2

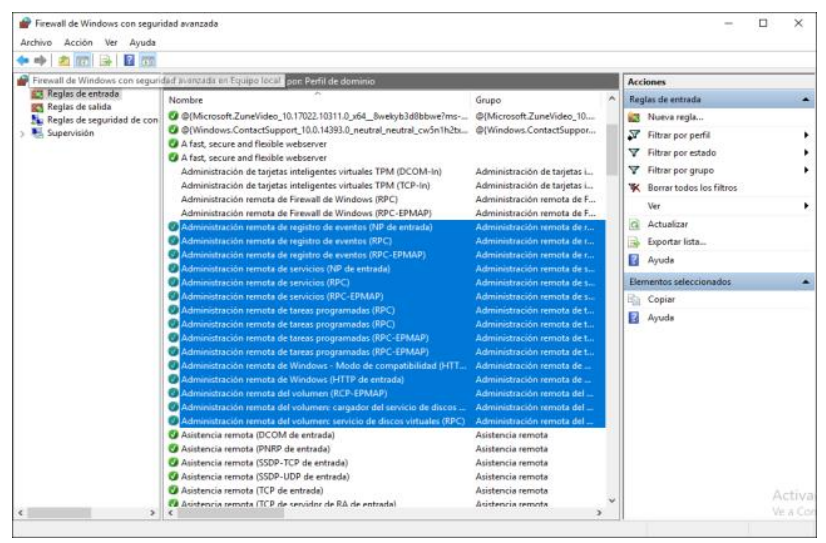

Figura 6, Reglas del Firewall de Windows 3

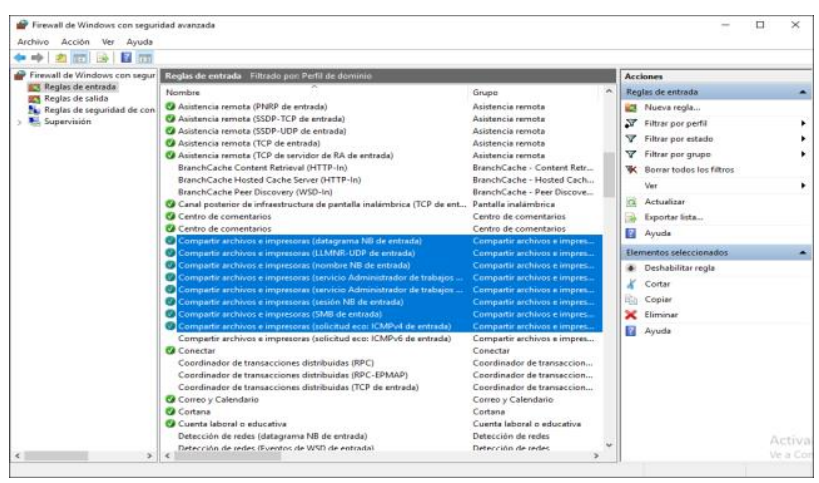

Figura 7, Reglas del Firewall de Windows 4

ALMANZA-GÓMEZ, Angel Alejandro, CORTÉS-CARRILLO, Francisco, MOLINA-DE LA ROSA, Laura y GÓMEZ-VIDAL, Juán Elí. Implementación de catálogo de servicios y servidor KACE. Revista de Ciencias de la Educación. 2019 


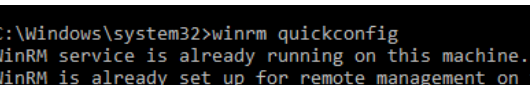

Figura 8, Activar Servicio de Administración Remota

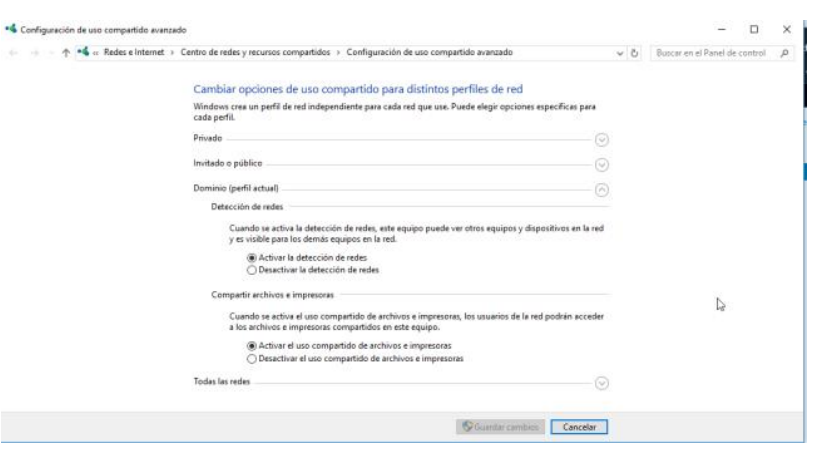

Figura 9, Activar la detección de redes y el uso compartido de archivos e impresoras

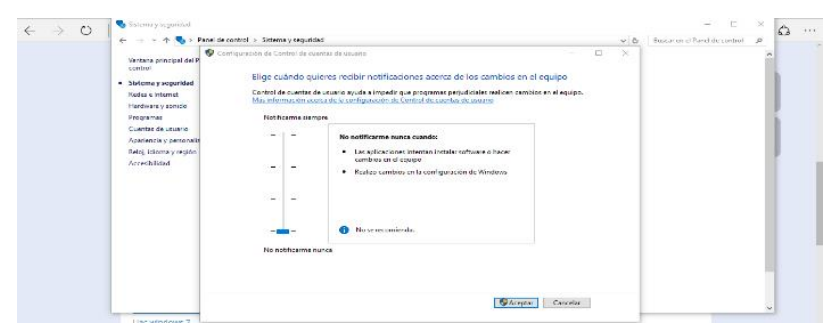

Figura 10, Deshabilitar UAC

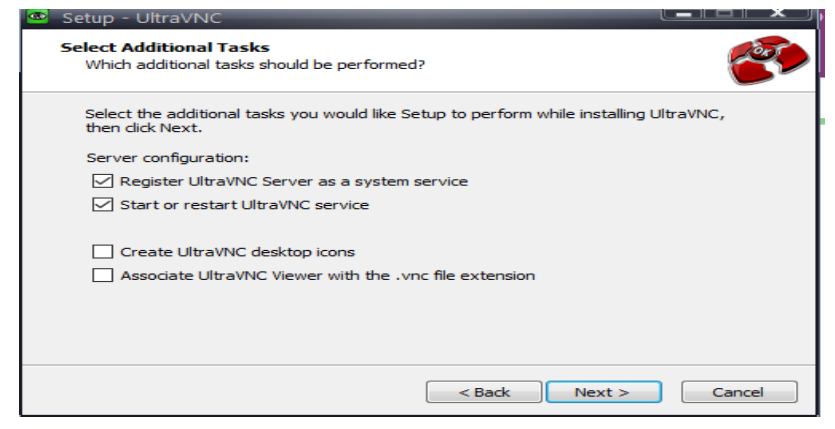

Figura 11, Instalación de VNC 1

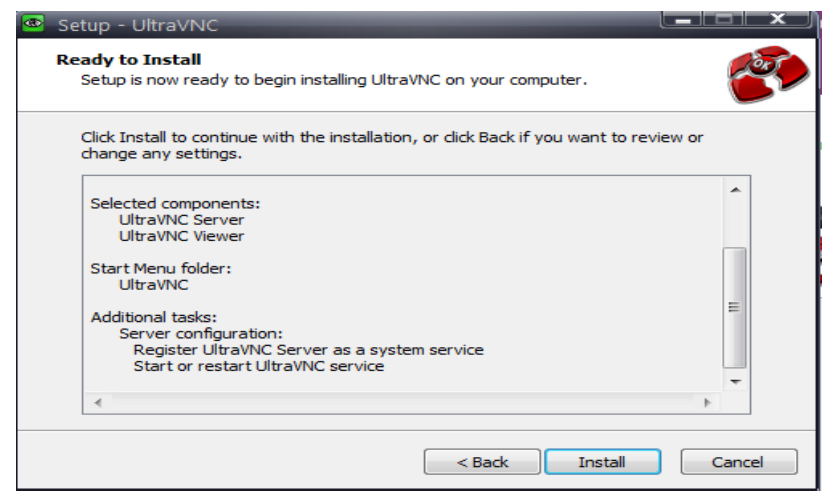

Figura 12, Instalación u VNC 2

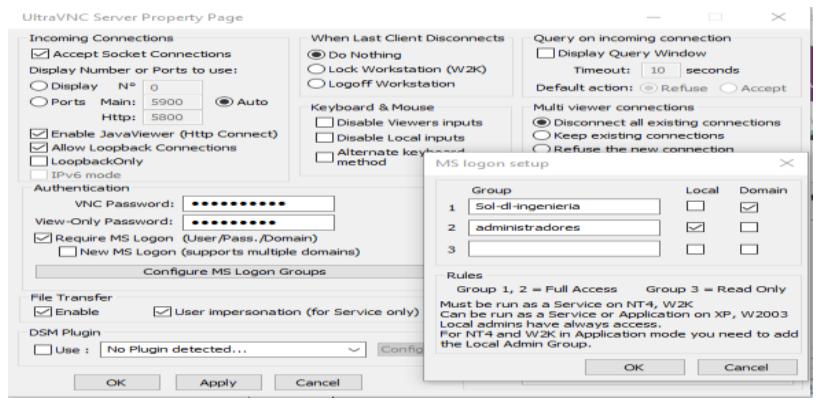

Figura 13, Instalación u VNC 3

Anexo 1

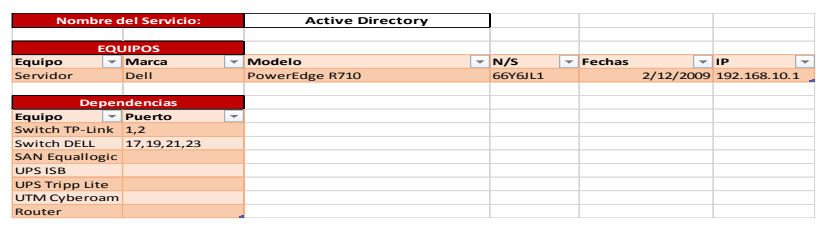

Figura 14, Servicio Active Directory

Anexo 2

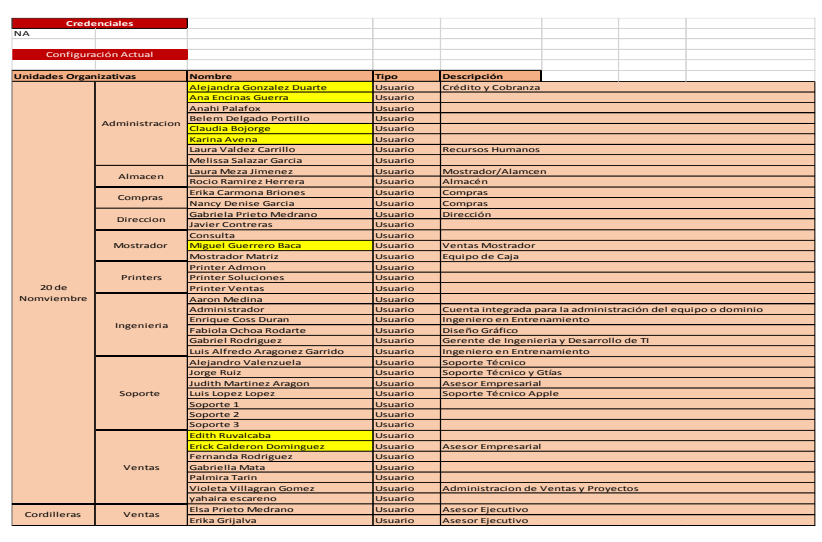

Figura 15, Configuración Active Directory

Anexo 3

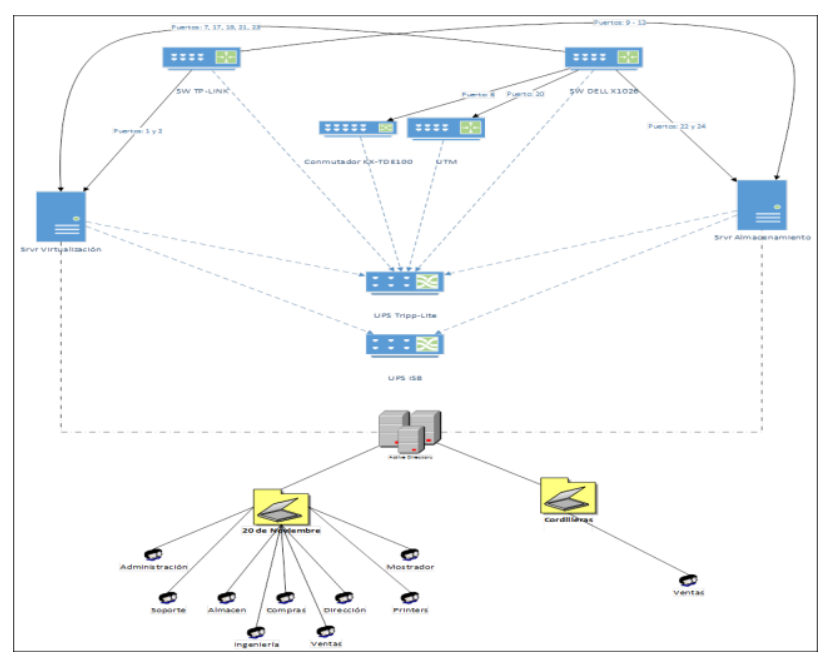

Figura 16 Diagrama del Servicio Active Directory 
Anexo 4

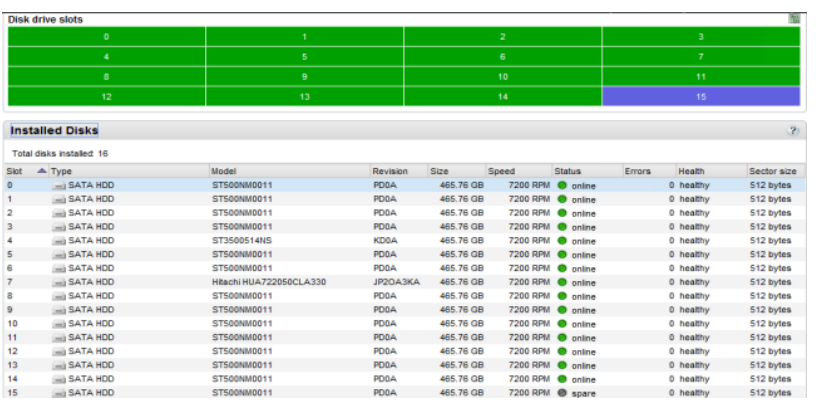

Figura 17, Configuración Almacenamiento 1

Anexo 5

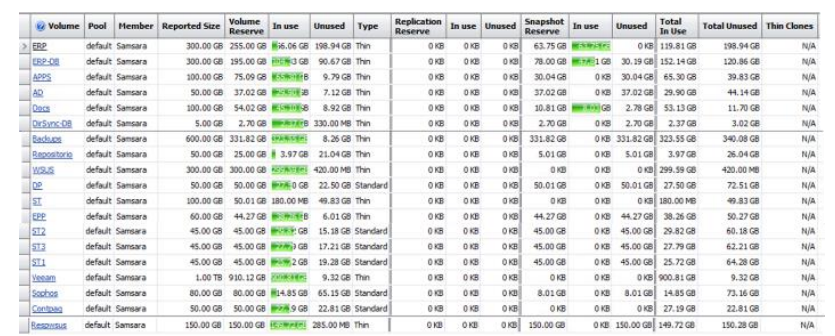

Figura 18, Configuración Almacenamiento 2

Anexo 6

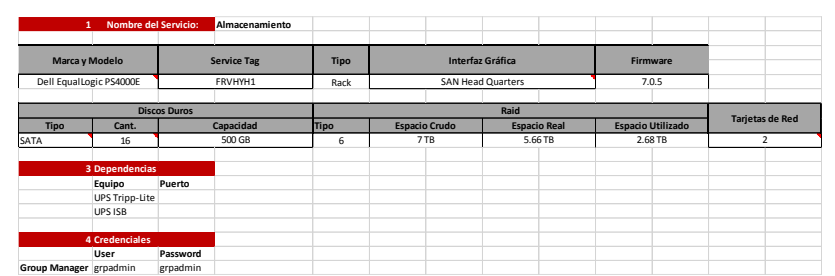

Figura 19 , Servicio Almacenamiento

\section{Anexo 7}

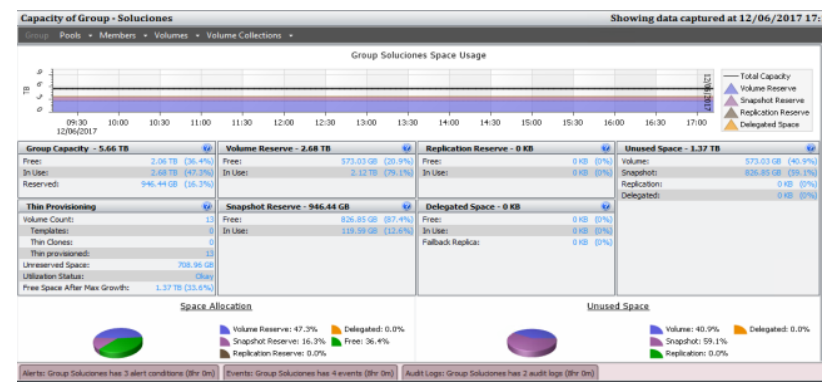

Figura 20, Configuración Almacenamiento 3

Anexo 8

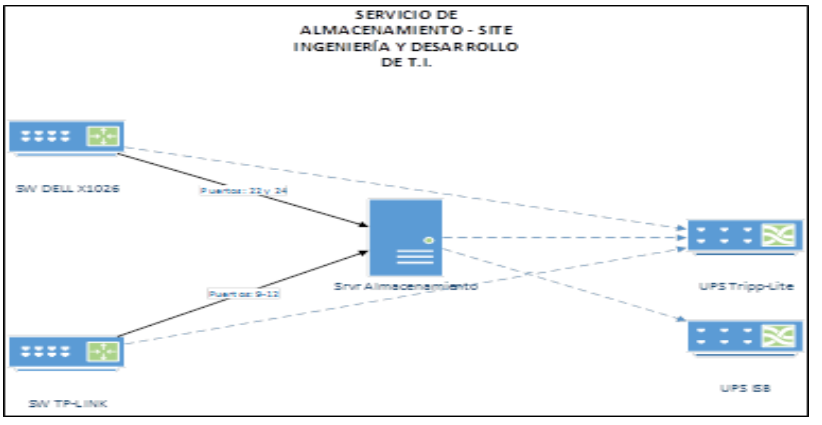

Figura 21, Diagrama Almacenamiento

ISSN: 2523-2436

ECORFAN® Todos los derechos reservados
Anexo 9

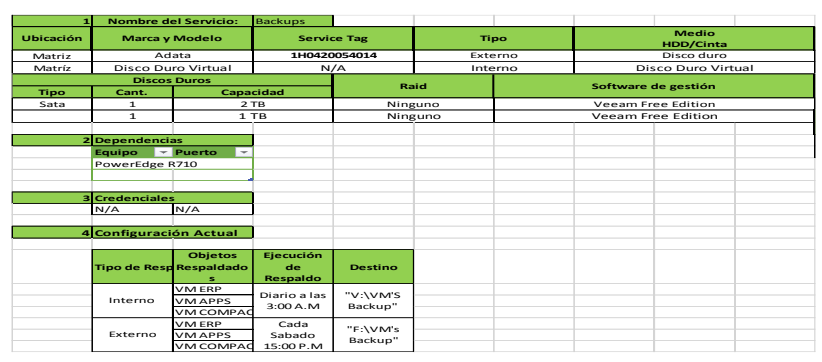

Figura 22, Servicio Backup

Anexo 10

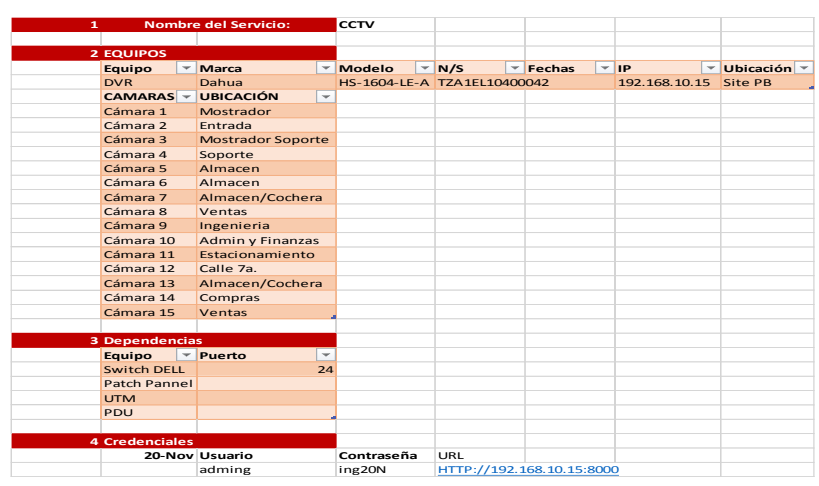

Figura 23, Servicio CCTV

Anexo 11

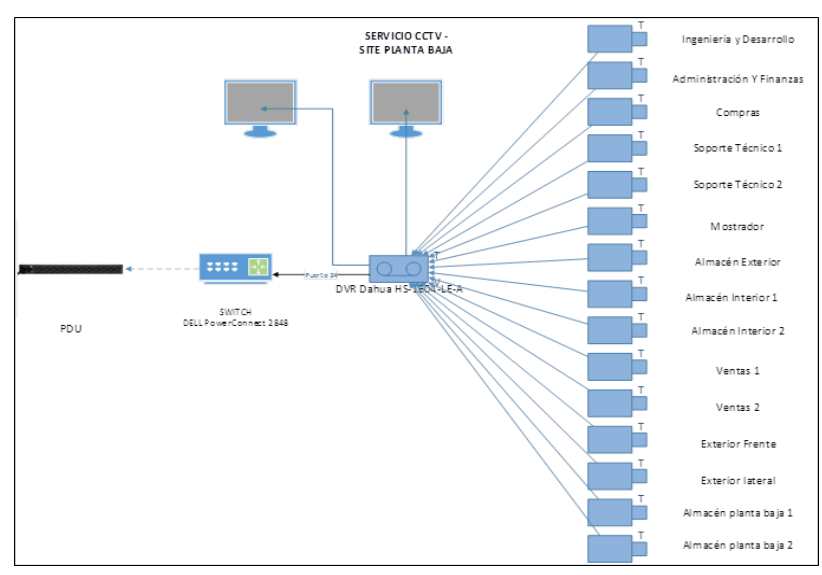

Figura 24, Diagrama CCTV

Anexo 12

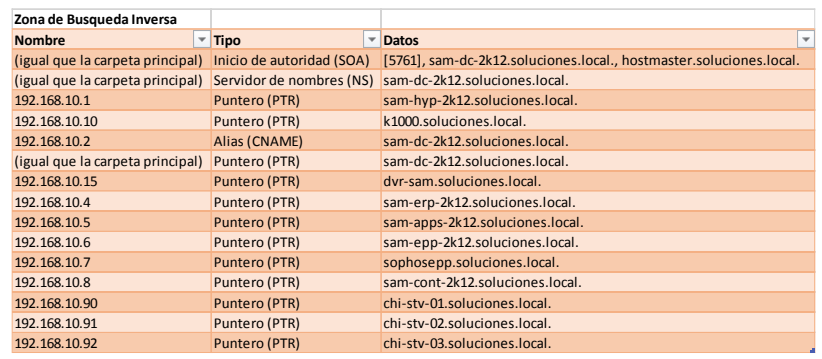

Figura 25, Servicio DNS 1 
Anexo 13

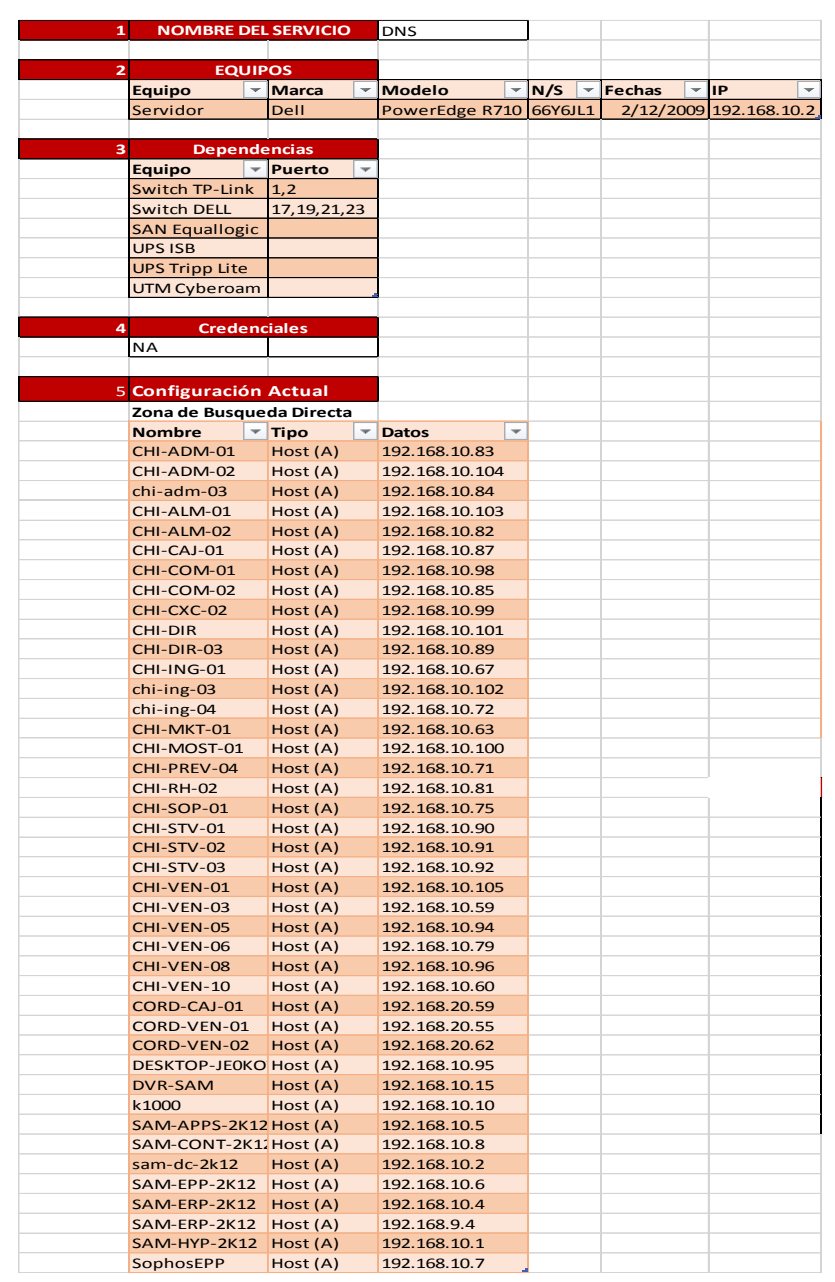

Figura 26, Servicio DNS 2

Anexo 14

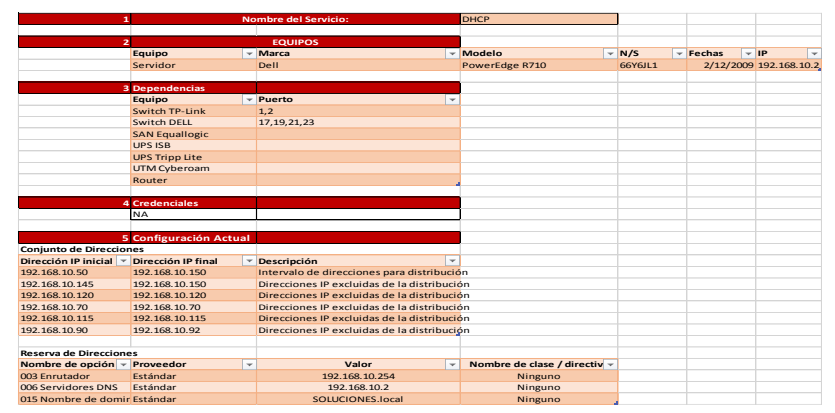

Figura 27, Servicio DHCP

Anexo 15

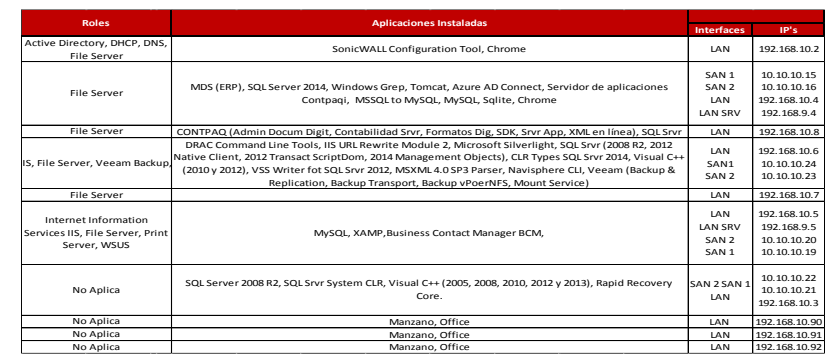

Figura 28, Servicio Hipervisor
Anexo 16

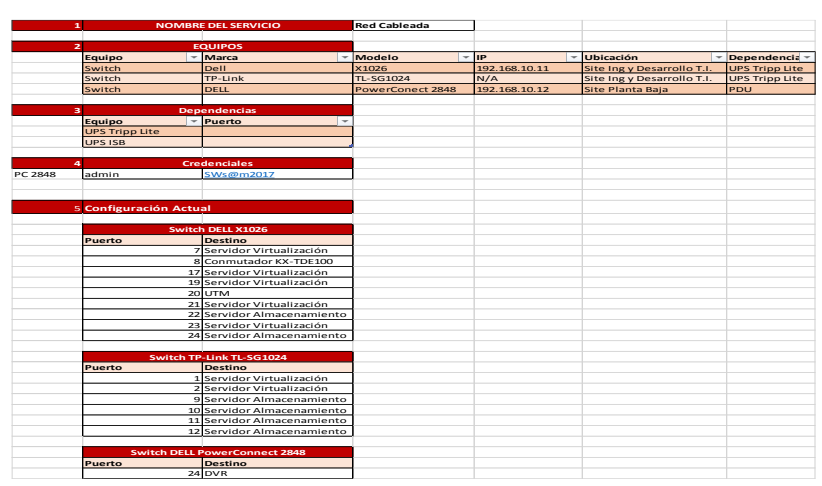

Figura 29, Servicio Red Cableada

Anexo 17

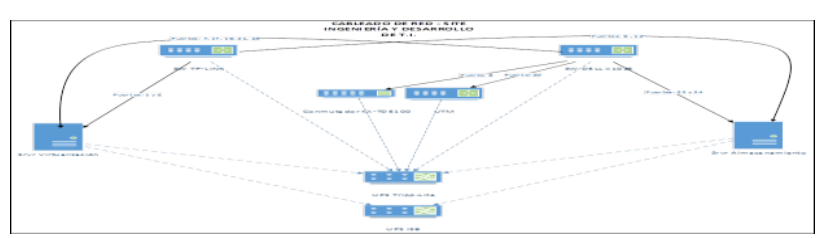

Figura 30, Diagrama Red Cableada

Anexo 18

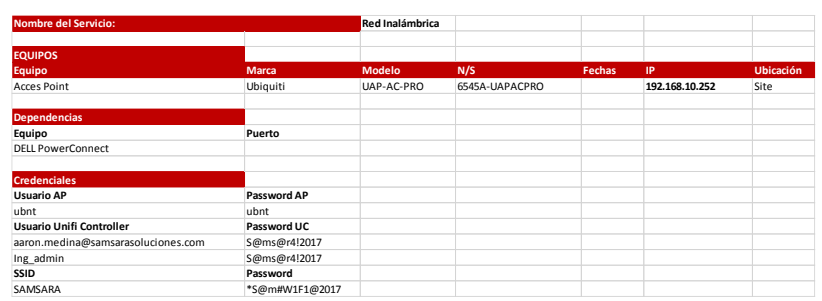

Figura 31, Servicio Red Inalámbrica

Anexo 19

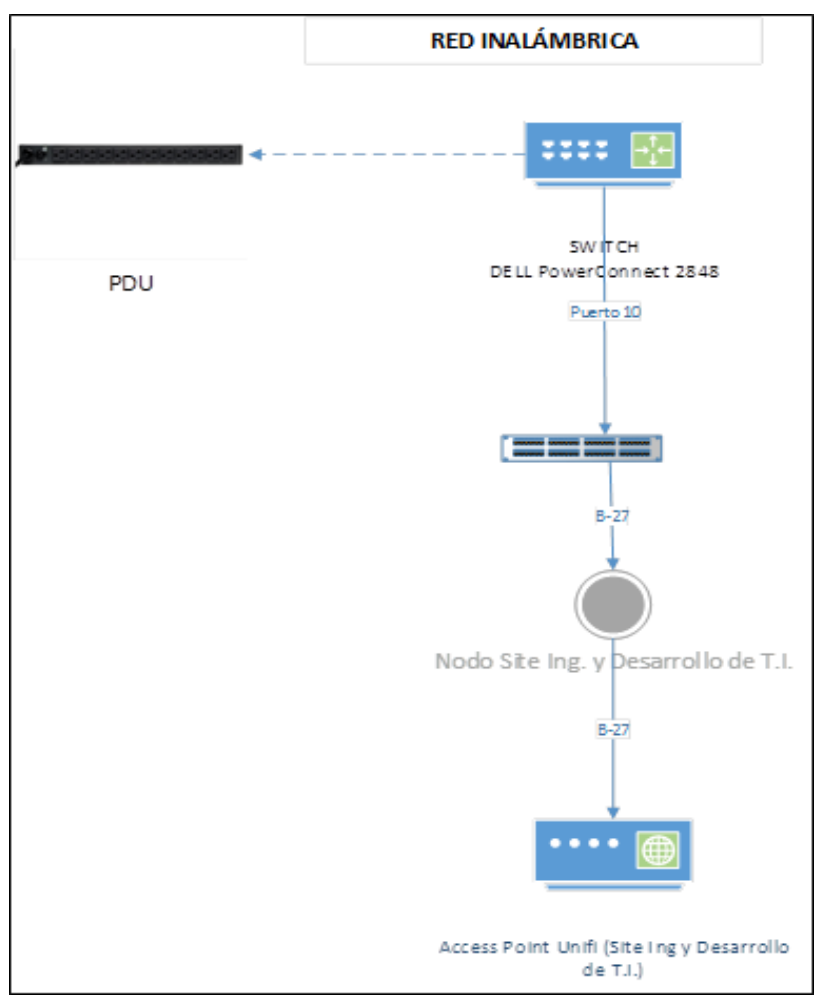

Figura 32, Diagrama de Red Inalámbrica

ALMANZA-GÓMEZ, Angel Alejandro, CORTÉS-CARRILLO, Francisco, MOLINA-DE LA ROSA, Laura y GÓMEZ-VIDAL, Juán Elí Implementación de catálogo de servicios y servidor KACE. Revista de Ciencias de la Educación. 2019 